\title{
Irritationen im Zwangskontext - Interaktionen im SGB II
}

Im Handlungsfeld der Beschäftigungsförderung justiert sich seit dem Inkrafttreten des SGB II das Verhältnis von Hilfe zu Kontrolle neu. Zudem wird der persönlichen Beratung und Hilfe Vorrang gegenüber der materiellen Versorgung eingeräumt. Die Eigenbemühungen der Nutzer zur Erwerbsarbeitsintegration rücken dabei in den Vordergrund. Im Fall „fehlender Mitwirkung“ steht den Fachkräften ein Arsenal an Strafmitteln zur Verfügung. Dieses Prinzip des „Förderns und Forderns“ schlägt sich auch in der Beratungsarbeit der Jobcenter - wenn auch nicht unbedingt programmgemäß - nieder. Wie dies geschieht, welche Muster sich dabei zeigen und welche Anforderungen und Zumutungen sich dadurch für die Beteiligten ergeben, skizziert dieser Artikel.

CHRISTIAN KOLBE

\section{Einleitung}

Im Handlungsfeld Soziale Dienstleistungen am Arbeitsmarkt könne man, so Ruth Enggruber (2010, S. 13), die Charakteristika des aktivierenden Sozialstaates „wie unter einem Brennglas“ beobachten. Die Reformen der vergangenen sieben Jahre nach Inkrafttreten des SGB II (Sozialgesetzbuch, Zweites Buch) tragen tatsächlich von Beginn an die ganze Mehrdeutigkeit des Aktivierungsparadigmas in sich. Für die Nutzerinnen und Nutzer sind dabei ebenso die Möglichkeiten von Partizipation oder Empowerment enthalten, wie auch die disziplinierenden Ausschließungsszenarien durch die Androhung und Durchführung von Sanktionen seitens der Behörde bei fehlender Mitwirkung. Besonders durch die Neujustierung des Schwerpunktes von der materiellen zur persönlichen Hilfe im SGB II rückt das Verhältnis zwischen den Akteuren in den Beratungsräumen stärker in den Vordergrund. Aus der für den Beratungserfolg notwendigen Koproduktion in personenbezogenen Dienstleistungen einerseits und dem als Zwangskontext ausgerichteten Handlungsfeld andererseits ergibt sich ein Spannungsverhältnis, das die professionellen „Aktivierer“ und ihre Klientel alltäglich bearbeiten. Der Ausgang dieser Aushandlungen ist dabei keineswegs entschieden.

Der Beitrag beschäftigt sich mit verschiedenen Ebenen dieser Aktivierungsthematik im Handlungsfeld Beschäftigungsförderung. Nach einer Skizze der Aktivierungspro- grammatik im Handlungsfeld (Abschnitt 2) und der Beschreibung irritierender organisatorischer Widersprüche darin (3) stehen Beratungsbeobachtungen im Zentrum des Textes (4). Das Schlusskapitel (5) fasst die Beobachtungen zusammen und setzt sie in Beziehung zu jüngeren Reformen.

Am Beispiel dreier Beratungsanalysen aus einem Evaluationsprojekt (ISR/infas/WZB 2008) werden Muster der Interaktion sowie der Bearbeitungs- bzw. Bewältigungsformen der Beteiligten rekonstruiert, um das darin gespielte mehrdeutige Spiel aus „Fördern und Fordern“ zu verstehen. Für die „Kundschaft“ steht dahinter die Frage, wie individuelle Bedürfnisinterpretationen (selbst) behauptet werden können und worin die Strategien in den Interaktionen für die Nutzerinnen und Nutzer liegen (können).

\section{Entwicklungen der Programmatik und der organisatorischen Logiken}

Auf den ersten Blick findet sich kaum Ungewisses im Handlungsfeld des SGB II, denn die Entwicklungen der vergangenen sieben Jahre nach Inkrafttreten des Gesetzes weisen für den Bereich der Beschäftigungsförderung eine kontinuierliche Engführung auf einen Teil des gesetzlichen Auftrags aus: Integration in den Arbeitsmarkt. 
Zudem erscheint der Prozess der Fallbearbeitung bezogen auf die direkten personenbezogenen Dienstleistungen hermetisch und hoch standardisiert gesteuert. Die Vehemenz, mit der die zentralen Akteure im Handlungsfeld, die Bundesagentur für Arbeit sowie das Bundesministerium für Arbeit und Soziales, in die Struktur der Einzelorganisationen und die Ausgestaltung des Leistungsprozesses intervenieren, lässt den Gedanken an jegliche - auch nur begrenzte - Form von Selbstbestimmung oder Autonomie in den Hintergrund treten. Die Entwicklungen der institutionellen Vorgaben (sei es als einheitliche PC-Fachanwendung oder als Handlungsempfehlung/Geschäftsanweisung [HeGa] der Zentrale der BA) erwecken gegenwärtig den Eindruck, dass sich die Ungewissheit der Anfangszeit nach Inkrafttreten des Gesetzes inzwischen sortiert hat. Dies zeigt sich auf verschiedenen Ebenen der „Wohlfahrtsproduktion“ (Kaufmann 2005):

- innerorganisatorisch durch die Etablierung vielfältiger Interventionen der zentralen Akteure (u. a. Controlling, Weisungen, Leitfäden);

- interorganisatorisch durch den Einzug neuer Rahmenbedingungen zur Kooperation zwischen Kosten- und Leistungsträger (u. a. Zuwendungsrecht vs. Vergaberecht; zentralisierter Einkauf von Leistungen; leistungsbezogene Vertragswerke und Zielkorridore);

- fallbezogen durch die einheitliche Nutzung von Profilingverfahren, Kundensegmentierung und leistungsbezogene Vertragswerke (Eingliederungsvereinbarung).

Wohlfahrtsproduktion im aktivierenden Sozialstaat zeigt sich aufgrund der Programmatik und Anlage im Handlungsfeld der Beschäftigungspolitik in spezifischer Weise: stark vereinheitlicht und arbeitszentriert, aber wenig pluralisiert und wenig an der Komplexität der Lebenssituation der zu Beratenden ausgerichtet.

Sieht man sich besonders die Zuspitzung der Fallbearbeitung auf das Ziel Integration in Arbeit im SGB II an, so geraten die eingangs erwähnten Desiderate der Partizipation für die Nutzerinnen und Nutzer sozialer Dienstleistungen in diesem Handlungsfeld bereits programmatisch zu einem Mythos. Vielmehr beschränkt sich die Entscheidung für sie vielfach darauf, sich auf die institutionellen Codes einzulassen oder aber wegen fehlender Mitwirkung sanktioniert zu werden. Unter diesen Bedingungen wird die Anrufung „Seid Subjekte!“ zu einer zwangsweisen Auf- und Anforderung an die eigene Selbstsorge, sich auf die Bedingungen des Arbeitsmarktes vorzubereiten und beschäftigungsfähig zu werden, um gegebenenfalls Zugang zum ersten, zweiten oder gar dritten Arbeitsmarkt zu erhalten. Ermächtigung durch die „Bereitstellung einer sozialen Infrastruktur“ (Hirsch/Steinert 2010) sieht diese Konstellation nicht vor. Im Gegenteil: Die immer detaillierteren Verfahren der Fallbearbeitung werden in wachsendem Maß auf die individuelle Fähigkeit, Verfügbarkeit und Willigkeit hin zugeschnitten, Risiken also privatisiert. Dass es gleichwohl unterhalb dieser personalisierenden Zuschreibungspraxis Formen der Bearbeitung gibt, die zwar nicht ausgreifende Ermächtigung verheißen, die aber häufig anderen als der programmatisch angedachten Ordnung geschuldet sind, veranschaulicht der Beitrag.

\section{Irritierende Aspekte bei der Implementierung in die Praxis}

Zieht man also allein die Programmatik und die beschriebenen Instrumente der verschiedenen Ebenen zurate, erscheinen die Wahlmöglichkeiten sowohl der Organisationen als auch der Fachkräfte durchaus eingeschränkt. Gleiches gilt für die Variationsmöglichkeiten der Interpretation oder Inanspruchnahme durch die Nutzerinnen und Nutzer. Bezogen auf die Frage der Vielfalt an Varianten der Umsetzung des SGB II wurden unterhalb der Programmatik und der Instrumente drei Spezifika ausgewählt, die die vermeintliche Abgeschlossenheit des Prozesses irritieren:

\subsection{Gebrochene Organisationspfade}

Die Dynamik, mit der das Gesetz (weiter) entwickelt wurde und wird, mündet u. a. für die an der konkreten Dienstleistung beteiligten Akteure (Fachkräfte/Nutzerinnen und Nutzer) in einer erheblichen Verunsicherung. Neben der im SGB II angelegten doppelten Zielorientierung auf Grundsicherung und Integration in den Arbeitsmarkt beinhaltet die Einführung dieses Gesetzes die Zusammenlegung zweier lokaler Großverwaltungen. Diese erhebliche Umstellung führt dazu, dass die Organisationen mit ihren je eigenen Verwaltungskulturen eine neue Organisation gründen oder aber doch zumindest eine enge Kooperation implementieren sollen. Die Aushandlungsprozesse zwischen den Organisationen bilden auch sieben Jahre nach Inkrafttreten des Gesetzes die fragile Grundlage, auf der beraten, unterstützt, betreut, informiert, bestraft und gedroht wird. Auch wenn die Entwicklungen auf eine zunehmend arbeitszentrierte Zieljustierung hindeuten, so sind andere Interpretationen des Gesetzes weiterhin denkbar (vgl. ISR/infas/WZB 2008, S. 282f.).

\subsection{Machtzuwachs und Verunsicherung der Fachkräfte}

Die Fachkräfte entwickeln in ihrem Arbeitsalltag zwar von den Organisationen strukturierte, gleichwohl zu einem hohen Anteil eigenmächtige Arbeitsweisen und Strategien (vgl. Lipsky 1980). Vor dem Hintergrund der Betonung persönlicher Hilfe, gepaart mit mehrdeutigen Arbeitsanforderungen zwischen sozialer Stabilisierung und Arbeitsmarktintegration sowie zwischen standardisierten Dokumentationsanforderungen und Anru- 
fungen an sozialarbeiterische Standards, erlangen sie zusätzliche Machtbefugnisse und Handlungsoptionen einerseits und Verunsicherungen aufgrund fehlender oder widersprüchlicher Orientierungen andererseits. Zur Bewältigung wird in der Regel auf die Möglichkeiten des Einsatzes standardisierender Instrumente sowie auf das jeweils zur Verfügung stehende Professionswissen rekurriert, um den neuen Anforderungen gerecht zu werden (Kolbe 2011). Dazu zählen Creamingverfahren, ${ }^{\bullet}$ mit deren Hilfe die Fachkräfte das eigene Arbeitsaufkommen „steuern“ (Baethge-Kinsky et al. 2006), ebenso wie die Möglichkeit, Themen auszusparen (vgl. Brodkin 1997 „Kultur des Wegsehens"), um anderswo Kapazitäten für eine intensive Unterstützung zur Verfügung zu haben. Wie dies jeweils geschieht, richtet sich nach den spezifischen Bedingungen, die die Fachkräfte vorfinden und dem Professionswissen, mit dessen Hilfe sie ihre Arbeit interpretieren. Angesichts der sehr heterogenen Zusammensetzung der Fachkräfte - sei es, was ihre Ausbildung (Arbeitsvermittlung, Sozialarbeit, Verwaltung), ihre Berufserfahrung oder auch ihre (vorherige) Zugehörigkeit zu einer Behörde (Arbeitsagentur, kommunaler Sozialhilfeträger, freier Träger) angeht - sind die Lesarten des Auftrags entsprechend sehr unterschiedlich.

\subsection{Eigensinnige Interaktionsmuster und Arbeitsbündnisse}

Schließlich ist es die Interaktionssituation selbst, die in den Fallbearbeitungen eine ganz eigene Dynamik aufweist und in der die Ausgestaltung des Arbeitsbündnisses ${ }^{2}$ zwischen den Beteiligten stattfindet. Wenn auch von den Bedingungen im Zwangskontext perforiert, entstehen je spezifische Räume der Interpretation bzw. lassen sich auch im SGB II unterschiedliche Interaktionsmuster identifizieren. In der Regel werden dabei Teile des gesetzlichen Auftrags bearbeitet, andere Teile werden ausgespart. Auch für die Interaktionen lassen sich demnach Unschärfen erkennen, die auf den Prozess eines muddling through („Sich-Durchwursteln") in den Beratungen hindeuten. Die Eindeutigkeit der Programmatik verschwimmt spätestens dort, wo es um die Strategien und Taktiken von Nutzerinnen und Nutzern in den jeweiligen Beratungssituationen geht.

Aus dieser Gemengelage aus programmatischen Ansagen, institutionell bedingten Irritationen und eigenwilligen Handlungsmustern werden die Charakteristika der Interaktionen sowie darin die jeweiligen Perspektiven der Hilfeberechtigten analysiert. Diese erhalten insbesondere durch die veränderte Schwerpunktsetzung im SGB II, von materieller zu persönlicher Hilfe, eine prominenter werdende Stellung bei der Umsetzung des Gesetzes.

\section{Was geht hier eigentlich vor? - Beobachtete Interaktionen}

Interaktionen im SGB II sind inzwischen im Zuge der oben genannten Betonung persönlicher Hilfe in diversen Studien untersucht worden. Zuletzt konzentrierten sich beispielsweise Boehringer/Wolff(2010) in ihrem Aufsatz darauf, wie sich der PC in die Interaktionen zwischen Beratenden und Klientinnen und Klienten einschreibt. Schütz (2010) stellt einen Vergleich der Fallbearbeitungen im SGB II und SGB III an.

Mehr oder weniger deutlich formuliert zeigt sich in vielen Studien trotz unterschiedlicher Akzentsetzungen ein Konsens darüber, dass eine an den Nutzerinnen und Nutzern orientierte Fallbearbeitung in der Regel schwer möglich und eine gemeinsam ausgehandelte Dienstleitung nur selten zu erkennen ist. Für die Situation der Hilfeberechtigten zeichnen die diskutierten Studien einheitlich ein erheblich eingeschränktes Wahl-Szenario. Die Beobachtungen, die im Übrigen in vielfältiger Weise durch diverse internationale Studien - besonders der frühen Aktivierungsprogramme in den USA (vgl. u. a. Brodkin 1997; Meyers et al. 1998; Lurie 2006; Riccucci 2005), aber auch in europäischen Nachbarstaaten (vgl. u. a. Thorén 2008) - gestützt werden, weisen deutlich auf eine von der Organisation geprägte „Interaktionsordnung“" (Goffman 1994) hin. Die Aussage einer Beraterin einer US-amerikanischen Aktivierungsbehörde steht dabei sinnbildlich für die regelmäßig beobachtbare Grundlogik der Fallbearbeitungen: „You've got to talk them into something." (Brodkin 1997, S. 16).

Frühe Studien von Baethge-Kinsky et al. (2006/2007) und Kolbe/Reis (2005/2008) geben unterschiedliche Hinweise darauf, welche Faktoren einflussreich für die Fallbearbeitung im SGB II sind bzw. sein können. Nimmt man die Ergebnisse der Studien zusammen, so ergibt sich daraus ein vielfältiges „Wirkungsbündel“ (Maeder/Nadai 2004, S. 56): Danach wirken Standardisierungen massiv auf die Interaktionen ein, nicht aber zwingend in der programmatisch intendierten Weise. Vielmehr scheinen sie überformt von den Bedingungen in den Organisationen, von dem zur Verfügung stehenden Professionswissen der Fachkräfte und von den Pfaden und jeweiligen Vorgeschichten der Einzelorganisationen wie der Kooperationstraditionen. Die Studien legen ihr Hauptaugenmerk darauf, genau die programmatischen, organisationalen und professionellen Voraussetzungen $\mathrm{zu}$ bestimmen, die den Charakter der Interaktionen mitverantworten.

\footnotetext{
Im Sinne einer „Bestenauslese” derjenigen Personen, die erfolgversprechend und mit möglichst überschaubarer Arbeitsbelastung beraten werden können.

(2) Hier verstanden als die Verständigung über die geltenden Konventionen und Spielregeln in den behördlichen Beratungssituationen (vgl. dazu allgemeiner Steinert 1998).
} 
An die Analyse der vorangestellten Studien angelehnt interpretiere ich in diesem Abschnitt drei Gespräche in der Arbeitsvermittlung bzw. dem Fallmanagement. Selbstverständlich lassen sich im SGB II je nach Situation und Konstellation diverse Szenarien rekonstruieren, in denen andere als die ausgewählten Interaktionsmuster und Situationsbewältigungen (z. B. systematische Ausschlüsse bestimmter Zielgruppen, konfligierende Interaktionen usw.) auffindbar sind. Da die beobachtete Praxis in der Regel jedoch davon getragen ist, habitualisierte und konventionalisierte Kommunikationsformen nicht zu verlassen, weil die Beteiligten wissen, was auf dem Spiel steht, wurden Fallbearbeitungen ausgewählt, in denen ein grundsätzlicher Konsens über die Regeln der behördlichen Kommunikation vorherrscht. Die beobachteten und in Gruppen analysierten Gespräche fanden zu einem frühen Zeitpunkt der Fallbearbeitung (Erstgespräche) statt. Dabei wurden drei Männer im Alter von Mitte bis Ende 20 beraten, die sich bezüglich ihrer Arbeitsmarktnähe und ihrer implizit oder explizit geäußerten Wünsche und Vorstellungen für das Gespräch gravierend voneinander unterscheiden (Übersicht 1).

\subsection{Interaktionsmuster}

\subsubsection{Beraterdominanz versus schweigendes Zur-Kenntnis-Nehmen}

Obwohl die beobachteten Fälle unterschiedlich gelagert sind, ist für alle eine erhebliche Dominanz der Berater im Gespräch kennzeichnend. Die Rollen sind eindeutig verteilt. Das Deutungsmonopol des Beraters wird nur in einer Sequenz in Fall 1 unterlaufen, als der Klient unaufgefordert das Gespräch zusammenfasst und seine Perspektive noch einmal in den Vordergrund stellt. Die Dominanz betrifft sowohl die reinen Gesprächsanteile als auch die Entscheidungen darüber, was Gegenstand der Aushandlungen im Gespräch ist, wann zu einem anderen Thema übergegangen wird und wie darüber gesprochen wird. Dabei erweisen sich die verschiedenen Standardinstrumente (Bewerberprofil, PC-Fachanwendung insgesamt und die Eingliederungsvereinbarung) als besonders strukturunterstützend. Sie betonen die Dominanz dahingehend, dass sie Monologe befördern und den, teilweise durchaus zugewandten, Paternalismus verstärken. In den Gesprächssituationen hat dies zur Folge, dass die Berater den Eindruck vermitteln, wenig von den Personen wissen zu wollen, geschweige denn, welches Anliegen und welche Bedürfnisse die Person mit zum Termin bringt. Augenfällig wird dies bereits in den Eingangssequenzen, in denen den Hilfeberechtigten wenig Möglichkeit gegeben wird, ihre Anliegen vorzustellen, ihre Erwartungen und Ängste zu beschreiben oder gegebenenfalls ihre Wünsche $\mathrm{zu}$ formulieren.

Für den weiteren Gesprächsverlauf lässt sich beispielsweise in Fall 2 zeigen, dass Herr B in dem vollständig durch die Eingliederungsvereinbarung dominierten Gespräch in fast
ÜBERSICHT

\section{Ausgewählte Fallskizzen bei der Interaktion im SGB II*}

\section{Fallskizze 1 - Herr A}

Nach längerer, krankheitsbedingter Arbeitslosigkeit plant Herr A den beruflichen Wiedereinstieg Angesichts der fehlenden Ausbildung setzt er sich zunächst zum Ziel, sich kurzfristig als Hilfsarbeiter in verschiedenen, ihm bekannten Arbeitsbereichen zu bewerben. Durch seine Kenntnisse über die jeweiligen Arbeitsfelder ist er bestrebt, seine bestehenden Fähigkeiten durch verschiedene Bescheinigungen (wie z. B. Staplerschein) zertifizieren zu lassen, um auf dem Arbeitsmarkt eine günstigere Ausgangsposition innezuhaben. Dabei soll ihn das Jobcenter unterstützen.

\section{Fallskizze 2 - Herr B}

Herr B bezieht zurzeit aufstockende Leistungen nach dem SGB II. Er bewirbt sich nach kürzlich abgeschlossener pflegerischer Ausbildung auf Stellen im medizinischen Bereich in R. Langfristig ist es sein Ziel, ein Studium der Pflegewissenschaften zu absolvieren. Er hat dazu bereits Informationen gesammelt, sich an zwei Hochschulen beworben und wartet auf Nachricht.

\section{Fallskizze 3 - Herr C}

Bedingt durch einen Umzug aus privaten Gründen sucht Herr C im Raum R nach einem Ausbildungsplatz in der Malerbranche. Aufgrund der sich abzeichnenden Schwierigkeiten bei der Ausbildungsplatzsuche bewirbt er sich zugleich auf Hilfsarbeiterstellen in der von ihm favorisierten Branche.

* Zur Anonymisierung der Fälle wurden die Namen entfernt und die Fallgestalt leicht verändert.

Quelle: Zusammenstellung des Autors. der Hälfte der Gesprächseinsätze die Einlassungen des Beraters lediglich schweigend-zustimmend zur Kenntnis nimmt. Während der Berater zu Beginn des Gesprächs die berufliche Entwicklung und die eigenen Anstrengungen des Herrn B bei der Arbeitssuche abfragt und ihm für die Einträge in die PC-Maske zweckbezogen Darstellungsraum gewährt, wird das Gespräch im weiteren Verlauf zu einem Monolog über die Form und die Inhalte der Eingliederungsvereinbarung, einschließlich einer langen Sequenz über die Konsequenzen, die der Klient im Fall fehlender Mitwirkung zu tragen hat. Herrn B kommt in dieser Sequenz die Aufgabe zu, durch zustimmendes Nicken die Anforderungen zur Kenntnis zu nehmen. Die im vorangegangenen Teil des Gesprächs dargestellten ambitionierten und realistischen Pläne und Vorstellungen zur Integration in Arbeit bzw. zur Weiterqualifizierung spielen bei der Eingliederungsvereinbarung keine Rolle mehr. Stattdessen wird bei der Beschreibung der Rechtsfolgen ein fiktives Missbrauchsszenario entworfen, in dem der Berater detailliert die Chronologie der Bestrafungen im Fall von Verfehlungen aufführt, wenngleich alle Ausführungen zu den beruflichen Planungen des Herrn B darauf hinweisen, dass dieser so eigeninitiativ ist, dass von möglichen Sanktionen überhaupt keine Rede sein kann.

Ein anderes Instrument spielt in Fall 1 eine maßgebliche Rolle für die Fallbearbeitung. Die Erstellung des Bewerberprofils in der PC-Fachanwendung wird hier in doppelter Hinsicht zum zentralen Bearbeitungsmodus. Einerseits erhebt der Berater in einer standardisierten Abfrage berufskundliche Daten und steuert den Prozess dadurch auf die Integration in Erwerbsarbeit, und zwar auf ein be- 
stimmtes Segment des Arbeitsmarktes hin, obwohl Herr A durchaus andere Erfahrungen vorweist. Dabei tritt besonders die Engführung auf die unmittelbare Verwertbarkeit der Informationen für den Integrationsprozess in Arbeit in den Vordergrund. Was nicht erfolgt, ist die Bearbeitung der Fragen, die der Klient selbst anregt (Krankheit und Schulden), die Hinweise auf beantragte und nicht erstattete Bewerbungskosten und nicht zuletzt dessen ausdrücklich angesprochene Qualifizierungswünsche (Staplerschein), die er aufgrund seiner eigenen Berufserfahrung als sinnvoll in der Branche erachtet und anfragt. Der Berater begibt sich nicht aus dem Modus der berufskundlichen Abfrage heraus. Durch die Vorgaben der Fachanwendung gerät tendenziell aus dem Blick, was quer zu den institutionell als relevant definierten Themen für die Arbeitsintegration zu liegen scheint

\subsubsection{Standardschablonen versus besondere Lebenssituationen}

Alle Beispiele veranschaulichen, dass sich die Fallbearbeitung stark über die Anwendung der Standardinstrumente konturiert, die sich schablonenhaft auf viele Sequenzen der Fallbearbeitung legt. Die Eingliederungsvereinbarung wird zudem zum Ort autoritärer Setzungen, wenn angesprochene Themen nicht in die Vereinbarung einfließen (Staplerschein), während andere, die vorher nur kurz erwähnt werden (so z. B. eine Trainingsmaßnahme zur Berufsorientierung in Fall 2) dennoch aufgenommen werden. Auf diese Weise können Gespräche stattfinden, ohne dass näher auf die individuelle Situation eingegangen wird. Die teilweise explizit formulierten Unterstützungsvorstellungen bleiben dann unberücksichtigt.

Wie wenig die Bearbeitungsweisen auf die Lebenssituation hin ausgerichtet werden, zeigt sich in allen Fällen, graduell jedoch unterschiedlich. Teilweise kann dies als eine „Kultur des Wegsehens“ (vgl. Brodkin 1997) interpretiert werden, wenn sich die Berater nicht auf das beziehen, was die Klienten an biografischen Angeboten formulieren: In Fall 1 werden die verschiedenen Arbeitserfahrungen von Herrn A nicht in die Beratung integriert und auch die schwierige Ausgangssituation (Krankheit, Schulden, ohne Ausbildung) wird nicht zum Gegenstand der Beratung gemacht. In Fall 2 werden umgekehrt die erfolgreiche berufliche Laufbahn ebenso wie die systematischen Anstrengungen für eine nachhaltige Integration in Arbeit von Herrn B zwar zur Kenntnis genommen und wertgeschätzt, dies wird aber sowohl durch die angedrohten Sanktionen als auch durch die in der Eingliederungsvereinbarung festgelegte Trainingsmaßnahme zur Berufsorientierung konterkariert, in die der hinlänglich berufsorientierte Herr B einwilligt. In Fall 3 wird der Ausbildungswunsch von Herrn $\mathrm{C}$ zwar aufgegriffen, indem eine Empfehlung zur Verbesserung des Realschulabschlusses über einen Kurs an der Volkshochschule angeregt wird. Die von ihm selbst angesprochene (abgebrochene) Fachoberschule bleibt in der Folge jedoch unerwähnt.
Diese Formen fehlender oder eingeschränkter Berücksichtigung der jeweils individuellen Situation der Adressaten werden häufig von dem Verweis auf die verpflichtende Nutzung von Standardverfahren, Standardangeboten und der Aufforderung zu Eigenbemühungen flankiert. Damit werden Wege der Bewältigung der eigenen Arbeit gesucht und gefunden, sei es durch

- den Hinweis auf die Notwendigkeit des Erfüllens von Dokumentationspflichten bzw. die Rahmung des gesamten Gesprächs durch die Eingliederungsvereinbarung,

- das regelmäßige Weiterverweisen an einen Träger mit einem Strauß an standardisierten Maßnahmemodulen,

- den Verweis auf die Möglichkeit mit privaten Arbeitsvermittlern zu arbeiten und

- den Verweis auf die Notwendigkeit der im Aktivierungsprozess im Vordergrund stehenden Eigenbemühungen.

Bedingt durch diese entlastenden Delegationen bleiben die förderlichen Ankündigungen der Berater im Beratungsgespräch selbst insgesamt zurückhaltend. Entsprechend ihrer arbeitszentrierten Ausrichtung beraten diese eng entlang von berufskundlichen Fragen, während lebensweltliche Aspekte regelmäßig in den Hintergrund treten. Dies ist Ausdruck des Bestrebens der Berater, den verschiedenartigen Vorgaben und Erwartungen einigermaßen gerecht zu werden, ihr Pensum zu leisten und ihre Arbeit zu legitimieren. Dazu müssen sie eine Auswahl treffen, was zu tun ist (vgl. Lau/Wolff 1982).

\subsubsection{Vages Fördern versus verbindliches Fordern}

Die Hinweise auf Angebote sind in den beobachteten Gesprächen weder Ergebnis einer sorgfältigen Anamnese noch gemeinsamer Aushandlungen mit dem Klienten. Sie sind selten konkret und verbindlich. Vielfach erscheinen sie lediglich als Dokumentation der vorhandenen Kompetenz und Feldkenntnis der Berater, verbleiben aber in einem Präsentationsstatus. Die Berater verweisen auf die Realisierung der Pläne in der weiteren Fallbearbeitung. So nimmt der Berater in Fall 1 den Wunsch nach einer Zertifizierung der Staplerkenntnisse zwar auf, verweist aber lediglich allgemein darauf, dass dies wohl möglich sei, jedoch hinterlegt er diese vage Befürwortung nicht in der Eingliederungsvereinbarung. Dadurch belässt er es bei der Möglichkeit, beim nächsten Mal darüber noch einmal zu reden. Herr A erhält jedoch nicht die Gelegenheit, das Angebot durch den Eintrag in die Eingliederungsvereinbarung als Förderinstrument einzufordern. Dies kann durchaus als beispielgebend für den Umgang mit dem Instrument angesehen werden. Auf der Förderseite der Eingliederungsvereinbarung werden in den beobachteten Fällen lediglich Standardmaßnahmen eingetragen, die wenig Aufwand für die Berater nach sich ziehen.

Verbindlich und konkret werden die Hinweise der Berater in den untersuchten Gesprächen dagegen dann, wenn 
es um die Androhung von Sanktionen und die Verpflichtung auf den Nachweis regelmäßiger Eigenbemühungen geht. Hier endet das ansonsten stattfindende Abtasten zwischen den Akteuren. Es werden konkrete Zahlen festgelegt, die binnen Monatsfrist erreicht werden müssen; es werden die Varianten von Bewerbung benannt, die möglich sind, und ihre Abrechnungsmodi erläutert. Hier werden die Unterstützungsformen in Form finanzieller Kostenerstattung und das Verfahren der Erstattung ausführlich und konkret formuliert. Der Hinweis von Herrn A auf die fehlende Erstattung der Bewerbungskosten in vorangegangenen Verfahren bleibt allerdings unberücksichtigt.

\subsection{Situationsbearbeitungen}

Geht man von diesen Grundmustern der Interaktion in den Grundsicherungsstellen aus, so stellt sich die Frage danach, worin der (strategische) Umgang der Nutzer trotz dieser Vermeidungen besteht, um die eigene Lebenssituation angemessen einzubringen. Schaut man also auf deren Rolle in den untersuchten Gesprächen, verfügen diese insgesamt über eine in weiten Teilen des Gesprächs passive Rolle. Gleichwohl verhalten sie sich in den Situationen des Beratungssettings, treffen Entscheidungen. Dabei tauchen unterschiedliche Anpassungsformen auf, die für die eigene Nutzung der Situation in der Behörde wesentlich sind. Sei es als primäre Anpassungen im Sinne der Anerkennung behördlicher Zumutungen, oder aber auch Formen sekundärer Anpassungen, in denen die Nutzer informelle Wege zur Bewältigung der Situation unterhalb des vorgegebenen Rahmens suchen (vgl. Goffman 1973, S. 185ff.). Dies können spezifische Formen der Selbstinszenierung ebenso sein wie strategische Auslassungen bei der Darstellung bestimmter Bereiche des eigenen Lebens.

In den drei untersuchten Gesprächen verhalten sich alle Nutzerinnen und Nutzer so, dass sie im Spiel bleiben. Keiner steigt aus, wenngleich ihre Ausgangspositionen und die damit verknüpften Gesprächserwartungen erheblich voneinander abweichen. Während die Nutzer der Fälle 1 und 3 mit dem Termin durchaus konkrete Anliegen verbinden, sich Themen vorgenommen haben (Aus- oder Weiterbildung), ist die Ausgangssituation für Herrn B (Fall 2) eher ein Termin, der wahrgenommen werden muss, wenngleich keine Unterstützung hinsichtlich der eigenen Arbeitssuche zu erwarten ist. Der Termin ist ein notwendiges Übel, um aufstockende Leistungen zu erhalten.

Gleichgültig, mit welchem Anliegen die drei im Jobcenter ankommen, die Strategie, sich möglichst kooperativ und unauffällig zu verhalten, ist allen gemeinsam. Dazu gehört es, den Ausführungen zustimmend ohne Anmerkungen zu folgen, wenn die Berater die Möglichkeiten der Förderung und Anforderungen formulieren. Selbst konkrete Maßnahmen, die die eigene Planung aktiv behindern, werden, wie im Fall von Herrn B die Trainingsmaßnahme zur Berufsorientierung, ausdrücklich befürwortet. Auch auf das Zurückstellen des vom Klienten deutlich und mehrfach formulierten (einzig expliziten) Wunsches nach einer Zertifizierung seiner Staplerfahrfähigkeiten reagiert Herr A schließlich bei der Darstellung der Inhalte der Eingliederungsvereinbarung mit schweigender Zustimmung. Die Gespräche sind also geprägt durch ein zustimmendes Stillhalten der Nutzer, teilweise sogar auf Kosten der Anliegen, die für sie wesentlich sind. Auch wenn sie auf diese Weise keine Vorteile aus dem Verfahren ziehen und sich den üblichen Verfahren der Aktivierung vollständig unterziehen müssen (Eigenbemühungen nachweisen usw.), so werden sie zumindest nicht als Störer in der Organisation markiert.

Darüber hinaus besteht offenkundig Einigkeit darüber, was in den Sitzungen besprochen wird. Es geht um Erwerbsarbeit und es geht um Bereitschaft. Dies gilt auch für die Nutzer, die sich an die arbeitszentrierten Themen anlehnen, darin sachlich argumentieren und ihr Dabeisein bekunden. Alle präsentieren sich als ausgesprochen flexibel hinsichtlich der Wahl des Arbeitsplatzes und zeigen, was sie können. ${ }^{\circledR}$ Die Aktivitäts- bzw. Flexibilitätssignale werden auf unterschiedliche Weise zum Ausdruck gebracht, haben aber gleichwohl ihre Grenzen. In Fall 3 ist Herr C bereit, sich auch in anderen als den von ihm favorisierten Bereichen im Malergewerbe zu bewerben, nicht jedoch darüber hinaus. Außerdem zeigt er sich bereit, gleichzeitig an der Verbesserung seines Schulabschlusses zu arbeiten. In der Präsentation der beruflichen Planungen zeigt sich auch Herr B ausgesprochen aktiv. Er wartet gleich mit mehreren Strategien auf. Falls die langfristigen Pläne, einen Studienplatz zu bekommen, nicht sofort gelingen sollten, verfolgt er Bewerbungsstrategien zur kurz- und mittelfristigen Überbrückung der Erwerbslosigkeit. Schließlich präsentiert sich Herr C als universell einsetzbar und spielt das im Profiling vorgesehene „Einschätzspiel“ um die vorhandenen sozialen Kompetenzen bei der Erstellung des Bewerberprofils uneingeschränkt mit.

Zugleich verschwinden die eigenen Pläne nicht vollständig, d. h., zumindest ihre Vorstellungen von einer angemessenen Unterstützung ihrer eigenen Pläne scheinen in den Fällen 1 und 3 immer wieder auf. Aspekte ihrer Lebenssituation jenseits von Erwerbsarbeit, die nicht von den Beratern angefragt werden, werden umgekehrt von den Nutzern ebenso wenig als Beratungsthema eingefordert. Angesichts dessen, dass die Nutzer realisieren, was in der Situation besprochen wird und was aber auch nicht, entscheiden sie, bestimmte Themen (Schulden, Sucht usw.) nicht noch einmal anzusprechen bzw. zu einem späteren Zeitpunkt oder gar anderswo zu besprechen (vgl. Ludwig 2010), behalten sich also vor, nur Teile ihrer Lebenssituation zu präsentieren und wahren dadurch einen Rest ihrer Autonomie.

3 Vgl. "Zeigen, was man kann. Arbeitslose sollen sich und ihre Talente besser vermarkten", Süddeutsche Zeitung vom 25. Juli 2011. 
Die Codes nach denen gespielt wird, sind insgesamt - sei es, was die situative Anpassung an die bestehenden Machtverhältnisse im Jobcenter betrifft als auch hinsichtlich der Anpassungsbereitschaft an die Themenauswahl und deren Bearbeitungsformen - auch den Nutzern gut bekannt und werden in verschiedenen Spielarten bedient. Die beobachteten Bearbeitungsmuster sind „defensiv“ (Bareis/Cremer-Schäfer 2008), d. h. sie dienen dazu, Zugehörigkeit zu signalisieren, um „zu verhindern, abgehängt oder als ,überflüssig“ definiert zu werden“ (ebd., S. 129) und die Situationen aushaltbar zu machen. Sie sind aber nicht „,ausgreifend“ in dem Sinne, dass sich damit auch nur „begrenzte Autonomiegewinne“ (ebd., S. 118) realisieren ließen. Die Strategie lautet eher, auf die oben skizzierte Weise selbstbestimmt selektiv zu informieren.

\section{Schluss}

Den Gesprächsanalysen zufolge entziffern die Beteiligten die Anwendungsregeln des Spiels (vgl. Maeder/Nadai 2004, S. 78) sehr genau. Häufig erweist sich die Interaktion in der Beratungssituation als Schattenspiel, in dem sich die Beteiligten dahingehend verständigt zu haben scheinen, dem gemeinsamen Deutungsmuster Erwerbsarbeit (Ludwig-Mayerhofer et al. 2009, S. 172) eine entsprechende Inszenierung folgen zu lassen. Während die Nutzer sich in der Regel im Wissen um den Kontext, in dem sie sprechen, als aktiv, flexibel und bereitwillig in Bezug auf die Aufnahme einer Arbeit zeigen, schildern demgegenüber die Fachkräfte die potenziellen Möglichkeiten der Unterstützung, vielfach ohne dabei verbindliche Aussagen zu treffen oder genaue Perspektiven zu benennen. Im beiderseitigen Wissen um die Begrenztheit der Chancen auf Vermittlung in den ersten Arbeitsmarkt und der gleichzeitig notwendigen Betonung von der Wichtigkeit der Integration in diesen, verständigen sich die Parteien häufig darauf, zunächst ein teilweise wortreiches Stillhalteabkommen bestehend aus Kompetenzdemonstrationen und Bereitschaftsbekundungen hinsichtlich der Förderlogik zu schließen.

Von Beginn an nicht stillgehalten wird hinsichtlich der Darstellung des Straf- und Kontrollarsenals und des Einforderns von Eigenbemühungen. Bereits in diesem frühen Stadium sind die Fallbearbeitungen regelmäßig von Bedrohungsszenarien durch die Eingliederungsvereinbarung und die Rechtsfolgenbelehrung im Falle der fehlenden Mitwirkung begleitet, die den Zwangscharakter in Erinnerung rufen.

Aktivierung als eine Ermächtigung zur Bearbeitung schwieriger Lebenssituationen ist mit den Fallbearbeitungen für die Hilfeberechtigten selten verbunden. In den Beratungen findet diese teilweise instrumentell zweckgebunden an das Ziel der Integration in den Arbeitsmarkt statt. So soll in Fall 3 die Arbeit am Bewerberprofil die Funktion erfüllen, die Eigeninitiative von Herrn $\mathrm{C}$ zu aktivieren.

Die Anpassungsvarianten seitens der Nutzer konzentrieren sich darauf, möglichst unauffällig aus den Gesprächen herauszukommen und darin ein Mindestmaß an Selbstständigkeit hinsichtlich der Offenbarung ihrer Situation zu behaupten. Ihre Taktiken dienen dazu, (zunächst) möglichst wenig bei der Arbeit an einem gelingenden Alltag gestört zu werden. Eine aktive Inanspruchnahme behördlicher Strukturen zur selbstbestimmten Gestaltung der Lebenssituation findet dagegen nicht statt. Im besten Fall erhalten die Nutzer Standardunterstützungsleistungen, die entweder immer passen (z. B. Kostenerstattungen) oder sie werden mit der Hoffnung auf passgenaue
Angebote zu Bildungs- und Qualifizierungsträgern entlassen. Dies kann passen, basiert jedoch nicht regelmäßig auf der Grundlage einer sorgfältigen Anamnese oder gemeinsamer Aushandlung im Jobcenter.

Die jüngsten Entwicklungen geben kaum Anlass zu der Hoffnung, dass sich an den eingeschränkten Möglichkeiten für die Nutzerinnen und Nutzer etwas grundlegend ändern wird. Die aufgrund der Gesetzesänderung vom August 2010 auf zwei Formen reduzierte Trägerschaft zur Umsetzung des SGB II inklusive der damit verbundenen Vereinheitlichungen von Zielvereinbarungen für beide Trägerformen schränkt die Vielfalt der Varianten weiter ein. Darüber hinaus tragen die Neuorganisation der Maßnahmen mit einer stärkeren Ausrichtung an den arbeitsmarktzentrierten Zielen des Gesetzes (Instrumentenreform 2010; vgl. dazu Oschmiansky/Ebach 2009; Sell 2008) und nicht zuletzt die 2011 erfolgte Kappung der Mittel für Eingliederungsleistungen dazu bei, dass personenbezogene Dienstleistungen am Arbeitsmarkt inhaltliche Engführungen erfahren. Gepaart mit den multiplen und widersprüchlichen An- und Überforderungen an die Fachkräfte (Standardisierung und Professionalisierung zugleich; vgl. Kolbe 2011) verengen sich die Handlungskorridore für alle Beteiligten.

So beschränken sich die Möglichkeiten der Nutzerinnen und Nutzer lediglich darauf, zu entscheiden, ob sie als „würdige Leistungsberechtigte“ gelten wollen und sich für die ohnehin vorhandenen Angebote entschließen, oder ob sie sich diesen Angeboten verweigern. Auch wenn sich in dem stark bundesbehördlich-zentralistisch geprägten Setting durchaus lokale Interpretationen und Anwendungsregeln identifizieren lassen, ergeben sich daraus für die Nutzerinnen und Nutzer keinesfalls befreiende Möglichkeiten der Partizipation, vielmehr beschränkt sich die Modernisierung des Sozialstaates in diesem Handlungsfeld vielfach darauf, wenig anzubieten bzw. zu fördern und dafür umso mehr Pflichten in die Personen hinein zu verlagern bzw. von ihnen zu fordern.

\section{LITERATUR}

Baethge-Kinsky, V./Bartelheimer, P./Henke, J./Land, R./Willisch, A./Wolf, A. (2006): Neue soziale Dienstleistungen nach SGB II (Konzeptstudie). Forschungsbericht, Soziologisches Forschungsinstitut Göttingen (SOFI), vervielfältigtes Manuskript, http://www.sofi.uni-goettingen.de/fileadmin/ Peter_Bartelheimer/Material/ Neue_Dienste/IAB_NDL_Berichtsband.pdf (letzter Zugriff: 10.6.2008)

Baethge-Kinsky, V./ Bartelheimer, P./Henke, J./Wolf, A./ Land, R./ Willisch, A./ Kupka P. (2007): Neue soziale Dienstleistungen nach SGB II, Institut für Arbeitsmarkt und Berufsforschung, IAB-Forschungsbericht (15), Nürnberg Bareis, E./Cremer-Schäfer, H. (2008): Reproduktionsstrategien in Situationen der Armut und die Reproduktion von Armutsfeindlichkeit, in: Alisch, M./May, M. (Hrsg.): Kompetenzen im Sozialraum. Sozialraumentwicklung und -organisation als transdisziplinäres Projekt, Opladen, S. 85-107

Böhringer, D./Wolff, S. (2010): Der PC als „Partner" im institutionellen Gespräch, in: Zeitschrift für Soziologie 39 (3), S. 233-251

Brodkin, E. (1997): Inside the Welfare Contract: Discretion and Accountability in State Welfare Administration, in: Social Service Review 71 (1), S. 1-33 Enggruber, R. (2010): Professionelle Grundlagen Sozialer Arbeit für den Arbeitsmarkt, in: Dies./Burghardt, H. (Hrsg.): Soziale Dienstleistungen am Arbeitsmarkt in der professionellen Reflexion Sozialer Arbeit, Berlin, S. 13-59 Goffman, E. (1973): Asyle. Über die soziale Situation psychiatrischer Patienten und anderer Insassen, Frankfurt a. M.

Goffman, E. (1994): Die Interaktionsordnung, in: Ders.: Interaktion und Geschlecht, Frankfurt a. M., S. 50-104

Hirsch, J./Steinert, H. (2010): Sozialpolitik als Bereitstellung einer sozialen Infrastruktur, http://www.links-netz.de/K_texte/K_links-netz_sozpol.html (letzter Zugriff: 25.3.2011) 
Institut für Stadt- und Regionalentwicklung (ISR)/Institut für angewandte Sozialwissenschaft (infas)/Wissenschaftszentrum Berlin für Sozialforschung (WZB) (2008): Evaluation der Experimentierklausel nach § 6c SGB II - Vergleichende Evaluation des arbeitsmarktpolitischen Erfolgs der Modelle der Aufgabenwahrnehmung "Optierende Kommune“ und "Arbeitsgemeinschaft". Untersuchungsfeld 2: Implementations- und Governanceanalyse, Abschlussbericht Kaufmann, F.-X. (2012): Sozialpolitik und Sozialstaat. Soziologische Analysen, Wiesbaden

Kolbe, C. (2011): Geforderte Aktivierer. Fachkräfte im SGB II zwischen Ansprüchen und Bewältigungen, Frankfurt a. M.

Kolbe, C./Reis, C. (2005): Vom Case Management zum „Fallmanagement“. Zur Praxis in der Sozialhilfe und der kommunalen Beschäftigungspolitik am Vorabend von Hartz IV, Frankfurt a. M.

Kolbe, C./Reis, C. (2008): Die praktische Umsetzung des Fallmanagements nach dem SGB II. Eine empirische Studie, Frankfurt a. M.

Lau, T./Wolff, S. (1982): Wer bestimmt hier eigentlich, wer kompetent ist?, in: Müller, S. et al. (Hrsg.): Handlungskompetenz in der Sozialarbeit/Sozialpädagogik, Bielefeld, S. 261-302

Lipsky, M. (1980): Street-Level Bureaucracy. Dilemmas of the Individual in Public Services, New York

Ludwig, M. (2010): Local Area Networks (LAN): Die Organisation der kommunalen Eingliederungsleistungen, Vortrag im Rahmen derTagung "SGB 2.0“ der Ev. Akademie Loccum vom 24.-26. November, http://www.loccum.de/material/ arbeit/sgbii/ludwig.pdf. (letzter Zugriff: 3.1.2011)

Ludwig-Mayerhofer, W./Behrend, O./Sondermann, A. (2009): Auf der Suche nach der verlorenen Arbeit. Arbeitslose und Arbeitsvermittler im neuen Arbeitsmarktregime, Konstanz

Lurie, I. (2006): At the Front Lines of the Welfare System, A Perspective on the Decline in Welfare Caseloads, Albany

Maeder, C./Nadai, E. (2004): Organisierte Armut: Sozialhilfe aus wissenssoziologischer Sicht. Erfahrung - Wissen - Imagination, Konstanz

Meyers, M. K./Glaser, B./Mac Donald, K. (1998): In the front line of the welfare delivery: Are workers implementing policy reforms?, in: Journal of association for Public Policy analysis and Management 17 (1), S. 1-22

Oschmiansky, F./Ebach, M. (2009): Vom AFG 1969 zur Instrumentenreform 2009: Der Wandel des arbeitsmarktpolitischen Instrumentariums, in: Bothfeld, S./Sesselmeier, W./Bogedan, C. (Hrsg.): Arbeitsmarktpolitik in der sozialen Marktwirtschaft - vom Arbeitsförderungsgesetz zu Sozialgesetzbuch II und III, Wiesbaden, S. 79-93
Pfeiffer, S./Hacket, A./Ritter, T./Schütt, P. (2008): Arbeitsvermögen und Arbeitslosigkeit - Empirische und theoretische Ergebnisse der SGB-II-Evaluation, E-Paper, Institut für Sozialwissenschaftliche Forschung e. V, München, www.isf-muenchen.de/pdf/Arbeitsvermoegen_und_Arbeitslosigkeit.pdf Reis, C./Ludwig, M. (2011): Steuerungsillusionen und ihre praktischen Wirkungen. Das „Vier-Phasen-Modell“ der Bundesagentur für Arbeit als Lehrstück für Case Management, in: Case Management 8 (2), S. 67-77

Riccucci, N. M. (2005): How Management Matters: Street level Bureaucrats and Welfare Reform, Georgetown

Schütz, H. (2010): Zur Interaktion mit den Usern? Die Rolle und Einbeziehung von Kundinnen/Kunden bei der Vermittlungs- und Integrationsberatung. Vortrag im Rahmen derTagung "SGB 2.0“ der Ev. Akademie Loccum vom 24.-26. November, http://www.loccum.de/material/arbeit/sgbii/schuetz.pdf (letzter Zugriff: 3.1.2011)

Sell, S. (2008): Die schiefe Ebene der Standardisierung und Zentralisierung Argumente gegen einen Systemwechsel im SGB II durch den Gesetzentwurf zur Neuausrichtung der arbeitsmarktpolitischen Instrumente und alternative Lösungsansätze, Expertise für die LAG Arbeit Hessen, Remagener Beiträge zur aktuellen Sozialpolitik 03-2008, Remagen

Steinert, H. (1998): Kulturindustrie, Münster

Thorén, K. H. (2008): „Activation Policy in Action” - A Street-Level Study of Social Assistance in the Swedish Welfare State, Växjö

\section{AUTOR}

CHRISTIAN KOLBE, Dr., ist wissenschaftlicher Mitarbeiter am Institut für Stadtund Regionalentwicklung der Fachhochschule Frankfurt/M. und am Institut für Sozialpädagogik und Erwachsenenbildung der Goethe-Universität Frankfurt/M . Arbeitsschwerpunkte: Aktivierender Sozialstaat, Case Management, Professionalität Sozialer Arbeit.

cmkolbe@fb4.fh-frankfurt.de 